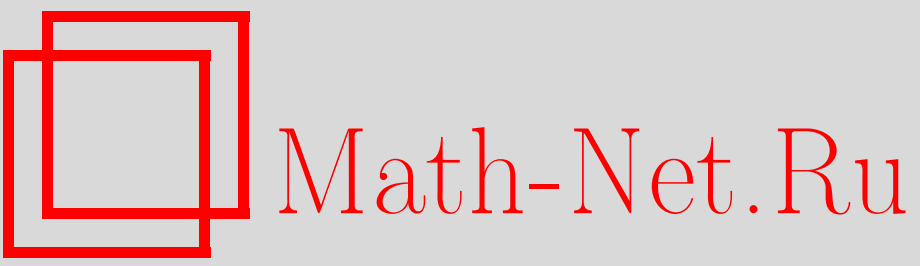

M. В. Дегтярев, Ограничение числа Пикара разрешения особенностей SL(2)многообразий Фано, УМH, 1996, том 51, выпуск 2, 155-156

DOI: https://doi.org/10.4213/rm953

Использование Общероссийского математического портала Math-Net.Ru подразумевает, что вы прочитали и согласны с пользовательским соглашением

http://www. mathnet.ru/rus/agreement

Параметры загрузки:

IP : 54.224 .135 .184

26 апреля 2023 г., 13:37:43 


\title{
ОГРАНИЧЕНИЕ ЧИСЛА ПИКАРА РАЗРЕШЕНИЯ ОСОБЕННОСТЕЙ SL(2)-МНОГООБРАЗИЙ ФАНО
}

\author{
М. В. ДЕГтярев
}

Пусть $X$ - трехмерное многообразие Фано с лог-терминальными особенностями, на котором бирегулярно действует группа $\mathrm{SL}(2, \mathbb{C})$ так, что имеется плотная орбита, эквивариантно изоморфная $\mathrm{SL}(2) / G$, где $G$ - конечная подгруппа в группе $\mathrm{SL}(2, \mathbb{C})$ и $G$ отлична от циклической (т.е. является бинарной группой икосаэдра, тетраэдра, октаэдра или диэдра).

ТеОремА. Для $X$ существует әквивариантное разрешение особенностей $\widetilde{X}$ такое, что $\rho(\widetilde{X}) \leqslant 7+16 / r(X)$, где $\rho$ - ранг группь Пикара, $r(X)$ - индекс Фано многообразия $X$.

ДокАЗАТЕльство. Приведем доказательство для случая, когда $G$ - бинарная группа икосаэдра, остальные случаи разбираются аналогично. Пусть $\varphi: \widetilde{X} \rightarrow X$ - морфизм разрешения особенностей на $X, \psi: \widetilde{X} \rightarrow Y$ - морфизм на единственное минимальное ( $\mathrm{SL}(2), G)$-многообразие $Y, Y=\overline{\mathrm{SL}(2) \cdot\left[f_{12}\right]}$ в $\mathbb{P}\left(R_{12}\right), f_{12}=f_{12}(x, y)=x y\left(x^{10}+11 x^{5} y^{5}+y^{10}\right)$ (см. [1]). Морфизм $\psi$ можно разложить в композицию раздутий с центрами в инвариантных кривых [1]: $\tilde{X}=Y_{n} \rightarrow Y_{n-1} \rightarrow \cdots \rightarrow Y_{3} \rightarrow Y_{2} \rightarrow Y_{1} \rightarrow Y_{0}=Y$. Решетка $N\left(Y_{i}\right)=\operatorname{Pic}\left(Y_{i}\right)$ свободно порождается классами неприводимых инвариантных дивизоров, которые являются рациональными линейчатыми поверхностями и, если $i \geqslant 3$, то они пересекаются трансверсально. Введем в $N\left(Y_{i}\right) \otimes \mathbb{R}$ произведение: положим $[D] *[E]=D \cdot e$, где $e-$ слой $E$, при этом, если $E \simeq \mathbb{P}^{1} \times \mathbb{P}^{1}$ с диагональным действием $\mathrm{SL}(2)$, то $e$ выбираем так, чтобы индекс пересечения $E \cdot e$ принимал наименшее значение, а если $E \simeq \mathbb{P}^{1} \times \mathbb{P}^{1}$ с действием на одной компоненте, то в качестве $e$ выбираем не инвариантный слой. Непосредственно проверяется, что введенная форма произведения симметрична и имеет сигнатуру $\left(1, \rho\left(Y_{i}\right)-1\right)$, т.е. она гиперболична. Сопоставим неприводимым инвариантным дивизорам естественным образом взвешенный граф $\Gamma\left(Y_{i}\right)$. Граф называется ланнеровским, если он гиперболичен, но любой его подграф̆ не гиперболичен (см. [2]). Покажем индукцией по $i$, что граф $\Gamma_{i}=\Gamma\left(Y_{i}\right)$ - ланнеровский. Так как $Y_{3}$ вместе с морфизмом $Y_{3} \rightarrow Y$ определено однозначно, то граф̆ $\Gamma_{3}$ имеет вид:

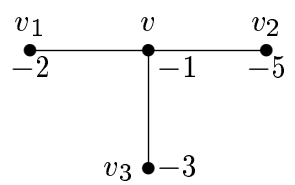

Вершины $v_{j}, j=1,2,3$, соответствуют дивизорам, изоморфным $\mathbb{P}^{1} \times \mathbb{P}^{1}$ с диагональным действием SL(2), вершина $v$ соответствует дивизору, изоморфиному $\mathbb{P}^{1} \times \mathbb{P}^{1}$ с действием на одной компоненте, поэтому на нем бесконечное число одномерных орбит. Так как при раздутиях ребер возникает дивизор, изоморфный $\mathbb{F} m, m>0$, мы можем считать, что морфизм $\sigma_{i}: Y_{i} \rightarrow Y_{i-1}$ раздувает ребра графа $\Gamma_{i-1}$ и собственный прообраз вершины $v$. Пусть $w$ - новая вершина графа $\Gamma_{i}$. Если $\sigma_{i}$ - раздутие ребра, то графф $\Gamma_{i}-w$ распадается на два непересекающихся графа, которые не гиперболичны по предположению индукции. Пусть $\sigma_{i}$ - раздутие собственного прообраза $\widetilde{v}$ вершины $v$. Граф $\Gamma_{i}-w$ отличается от $\Gamma_{i-1}$ уменьшением веса $\widetilde{v}$ на 1 . Стянем на $\Gamma_{i}-w$ все вершины с весом -1 . Этому будет соответствовать морфизм $\tau: Y_{i} \rightarrow Y_{4}^{\prime}$, где $Y_{4}^{\prime}$ - раздутие $Y_{3}$ в вершине $v$. Граф $\widetilde{\Gamma}=\Gamma\left(Y_{4}^{\prime}\right)-\tau(w)$ имеет вид:

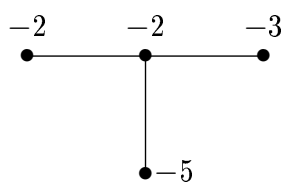

Работа выполнена при частичной поддержке Российского фонда фундаментальных исследований (грант № 93-011-1539), а также Международного научного фонда (грант № 90000). 
Он эллиптичен, т.е. имеет сигнатуру $(0,4)$ (см., например, [3]), но граф $\Gamma_{i}-w$ получается раздутием $\widetilde{\Gamma}$, следовательно, он также эллиптичен. Мы показали, что граф̆ $\Gamma(\widetilde{X})$ - ланнеровский. Так как $X$ - многообразие Фано, морфизм $\varphi$ стягивает все вершины с весом меньше -1 . Для оставшихся вершин $e_{j}$ определим DP-коэффициенты $\eta_{j}$ так же, как в работе [4]: $\eta_{j}=$ $-\left[\varphi^{*} K_{X}\right] *\left[e_{j}\right]=1+\sum_{i} \alpha_{i}\left[F_{i}\right] *\left[e_{j}\right]$, где $F_{i}$ - исключительные дивизоры морфизма $\varphi$, коэффициенты $\alpha_{i}$ определяются равенством $K_{\widetilde{X}}=\varphi^{*} K_{X}+\sum_{i} \alpha_{i} F_{i} ; \alpha_{i} \leqslant 0$, так как в качестве $\widetilde{X}$ мы можем взять терминальную модификацию $X$, которая неособа, поскольку изолированнье особенности $(\mathrm{SL}(2), G)$-многообразий не $\mathbb{Q}$-факториальны [5]. Если все $\eta_{j} \geqslant \varepsilon$, то говорят, что граф $\Gamma(\widetilde{X})$ удовлетворяет условию $\mathrm{DP}(\varepsilon)$. В этом случае по теореме 3.13 из [4] мы получаем оценку: $\rho(\tilde{X}) \leqslant 7+16 / \varepsilon$. Осталось заметить, что $r(X) \leqslant$ НОД $\left(\eta_{j}\right)$. Теорема доказана.

СлеДСтвиЕ. $\rho(\widetilde{X}) \leqslant 7+16 / k, k-$ индекс горенштейновости $X$.

Автор выражает благодарность Ю.Г. Прохорову за постановку задачи и внимание к работе, а также В. В. Никулину за полезные обсуждения.

\section{СПИСОК ЛИТЕРАТУРЫ}

[1] Mukai S., Umemura H. // Lect. Notes in Math. 1983. V. 1016. P. 490-518. [2] Никулин В. В. // Изв. АН СССР. Сер. матем. 1988. Т. 52. №6. С. 1032-1050. [3] Илиев А. Н. // Вестник МГУ. Сер. І. Матем., мех. 1986. №3. С. 38-44. [4] Алексеев В. А. // Изв. АН СССР. Сер. матем. 1988. Т. 52. №6. С. 1288-1304. [5] Крафот Х. Геометрические методы в теории инвариантов. М.: Мир, 1987.

Принято редколлегией 15.01.1996 